\title{
T. Lehtisalo
}

\section{$1887-1962$}

Von den Kindern des Gustaf Vilhelm Löfgrín, Volksschullehrer im Dorf Mustanoja in der Gemeinde Pöytyä, zeigte der älteste Sohn, der am 16. Juni 1887 geborene Toivo Vilho, bereits in seiner Schulzeit ein grosses Interesse an Forschungsreisen und an den finnisch-ugrischen Sprachen. Seine Lieblingsbücher waren u.a. "Elias Lönnrotin matkat" (Elias Lönnrots Reisen) und József Szinnyeis "Suomen kielen heimolaiset" (Die Stammverwandten des Finnischen). Als Oberschüler der sechsten Klasse besuchte er in den Sommerferien die nahen Stammverwandten der Finnen, die Esten in Muhu (Mohn) und Kuresaar (Arensburg) und freundete sich damals u.a. mit seinem Altersgenossen Willem Grünthal an. Im folgenden Frühjahr, im Mai 1906, änderte Toivo Löfgrén seinen Familiennamen in Lehtisalo und legte an der finnischen Oberschule zu Tampere privat das Abitur ab, ein Jahr früher als seine Turkuer Klassenkameraden.

Lehtisalo, gewandt im Schreiben, war zunächst — um sich Geld fürs Studium zu verdienen - ca. ein Jahr als Journalist in Turku tätig und ging dann im Herbst 1907 an die Universität von Helsinki. Seine Hauptfächer waren natürlich das Finnische und die finnisch-ugrischen Sprachen. Das Interesse an der Muttersprache beschränkte sich nicht auf das blosse Pflichtstudium, sondern Lehtisalo begann bereits in seiner Studentenzeit systematisch Aufzeichnungen aus dem Dialekt seines heimatlichen Kirchspiels zu machen, so dass er i.J. 1909 der Finnischen Literaturgesellschaft eine Dialektsammlung von 2250 Zetteln überreichen konnte. Im gleichen Jahr wurden in der Zeitschrift Virittäjä seine ersten, auf Material aus Pöytyä 
gründenden sprachwissenschaftlichen Artikel gedruckt: "Kansa hokuja laatimassa vieraista kielistäkin» (Das Volk schafft Wortspiele auch mit fremden Sprachen) und "Äännesubstitutsiota" (Lautsubstitutionsfälle). Lehtisalo betätigte sich melormals in den beiden folgenden Jahrzehnten weiter als Wörtersammler in seiner Heimat, zuletzt als Stipendiat der Wörterbuchstiftung 1924-1925. Damals wurde der Wortschatz von Pöytyä gründlich gesammelt; die Sammlung enthält an 31000 Zettel und ist immer noch besonders hinsichtlich ihrer phonetischen ind morphologischen Genauigkeit vorbildlich. In dieser Zeit entstand auch der Aufsatz mit dem heimatlichen Thema "Vanhoista kirkonpaikoista Pöytyällä paikannimien ja tarujen valossa" (Über alte Kirchenstellen in Pöytyä im Lichte von Ortsnamen und Sagen) (gedruckt 1928).

Die Probleme der finnischen Sprache standen Lehtisalo immer nahe, wenn er seine hauptsächliche Lebensarbeit auch auf einem ganz anderen Gebiete der Sprachwissenschaft vollzog. Er selbst schrieb über die Ausprägung seiner Laufbahn: "Ich fühlte [als Student] eine romantische Bindung an die verwandten Sprachen, und ausserdem hegte ich im Innern den Wunsch, nach Russland zu gelangen, um die finnisch-ugrischen Völker und ihr Leben näher kennenzulernen. Ich hatte an die Erforschung des Mordwinischen gedacht, aber da diese Sprache schon ziemlich gut bekannt war, wandte ich mein Hauptaugenmerk auf das Samojedische. Die samojedischen Sprachen waren bis dahin beinahe unbekannt. Nach Castrén war kein finnischer Sprachforscher bei den Samojeden gewesen. Mein Lehrer Prof. Heikki Paasonen begann gerade in dieser Zeit auf der Basis von Castréns knappem Material Vorlesungen über Samojedisch zu halten, und er bemerkte, das Aussenden von Forschungsreisenden zu den Samojeden sei notwendig. - I. J. 1910 wurde ich Kandidat und Magister und war mir unschlüssig darüber, was ich beginnen sollte. - - Da meinte der Wogulenforscher Artturi Kannisto, es sei nun an der Zeit, dass ich zu den Samojeden käme, und auf seine Aufforderung hin sprach ich darüber mit Professor E. N. Setälä. Dieser verschaffte mir auch sofort ein Stipendium für zwei Jahre und so begann meine Reise am Ende des Jahres 1910." Das Zitat 
stammt aus dem Vorwort von Lehtisalos Memoirenwerk "Tundralta ja taigasta" (Von Tundra und Taiga), das 1959 erschien. In diesem Buch erzählt der alte Forscher in ausgesprochen lustiger Form, was er vor einem halben Jahrhundert sah und erlebte, als er drei Jahre lang in Sibirien und Nord-Russland unter Juraksamojeden, Ostjaken, Syrjänen und Russen weilte. (Die Memoiren erschienen in deutscher Sprache schon i.J. 1957 als Vorwort von Lehtisalos »Juraksamojedischem Wörterbuch».)

Auf seiner ersten weiten Reise i.J. 1911 und 1912 erforschte Lehtisalo die Tundrajuraken. Anfangs wohnte er ungefähr ein Jahr an der Obmündung in dem grösseren Kirchdorf Obdorsk, von wo aus er kürzere Reisen in die Umgebung und eine Sommerfahrt an die Tas-Bucht unternahm. Dann ging er über den Ural zu den Juraken im ehemaligen Gouvernement Archangelsk. Die zweite Reise füllte das Jahr 1914 aus und ging zu den an den Nebenflüssen des $O b$, dem Ljamin, der Kiseljowskaja und Sachalinskaja, wohnenden Waldjuraken. - Der Forscher lernte die mürrischen, schwerfälligen Juraksamojeden gründlich kennen. Genau zeichnete er den Wortschatz und Sprachproben von über fünfzig Juraken aus verschiedenen Gegenden auf. Die meisten Sprachmeister waren verarmt, hatten ihre Rentiere durch eine Seuche verloren, und der Lohn. den ihnen der Fremde aus dem Auslande, der »lange Russe», für die leichte Sitzarbeit zahlte, war eine willkommene zusätzliche Einnahme. Mit der Zeit wurde jedoch der regelmässige Aufenthalt im Inneren und die »schriftliche Beschäftigung» für viele der Naturkinder unerträglich. Nur ganz wenige Samojeden begriffen irgendwie den Zweck einer derartigen Arbeit, doch zeigten sie zum Glück keinerlei Feindseligkeit dem Fremdling gegenüber, wie sie einige finnische Forschungsreisenden von Bewohnern Nordost-Russlands haben erfahren müssen. Die Waldjuraken waren zurückhaltender und scheuer als die Bewohner der Tundra. In einigen Gegenden flohen sie in die Wälder, unerreichbar für den Forscher. Ihrer Meinung nach sind nur die Samojeden, Ostjaken und Wogulen »richtige Menschen", weil sie den "gleichen Glauben" haben; alle anderen sind Herren, "Russen", die z.B. ganz hilflos im Gelände sind: "sie 
können nicht einmal von einem Dorf ins andere ohne abgesteckten Weg gehen". Zwischen den Juraken und den Obugriern herrschte wenigstens noch damals eine echte Blutsverwandtschaft, denn Eheschliessungen zwischen bestimmten jurakischen und obugrischen Geschlechtern waren gestattet und beliebt. - Lehtisalo sammelte auf seinen Reisen auch grosse Jengen sachrolkskundlich interessanter Gegenstände, die heute im Besitz des Nationalmuseums zu Helsinki sind, wie auch seine kürzlich gefundene Fotosammlung.

Das Arbeiten in der sibirischen Kälte und den elenden Hütten der Urbewohner war oft äusserst schwierig und anstrengend. zumal auch im Vaterland die Schwierigkeiten des isoliert schaffenden Forschers nicht immer begriffen wurden. Lehtisalo hat dem Verfasser dieser Zeilen über Einzelheiten seiner Forschungsmethoden und -prinzipien berichtet, die er in der Öffentlichkeit nicht berührt hat. Auch unter seinen nachgelassenen Papieren befinden sich einige Manuskriptbogen, die diese Umstände angehen und die wohl offensichtlich ursprünglich in der Vorrede zum juraksamojedischen Wörterbuch veröffentlicht werden sollten. - Da die tundrajurakischen Mundarten sich nicht in dem Masse unterscheiden wie z.B. die ustjakischen und wognlischen Dialekte, hiclt Lehtisalo es nur für nötig, das Obdorsker Tundrajurakische gründlich zu erforschen. In den anderen tundrajurakischen Gebieten hiclt er sich verhältnismässig kurze Zeit auf und begnügte sich häufig damit, lediglich die Lautstruktur und die lautlichen Besonderheiten dieser Dialekte kennenzulernen. Fand er jedoch jemanden, der sich gut auf die Volksdichtung verstand, schöpfte er dessen Schatz aus. Bei der Sammlung des Wortschatzes galt seine Hauptaufmerksamkeit den Verben, "da in ihnen und ihren Bedeutungen mehr interessanter Wechsel war als in den Benennungen der Gegenstände». Mitunter bedauerte er, dass er beim Sammeln nicht genügend Aufmerksamkeit auf einige wichtige Themenkreise gerichtet habe, z.B. auf die Verwandtschaftsbezeichnungen. Wegen der unerhört weiten Entfernungen und wegen unüberwindbarer Naturhindernisse - İberschwemmungen und schlechte Wegeverhältnisse - brauchte der Forscher verhältnismässig viel Zeit, wenn er in den sehr dünn besiedelten und 
fast weglosen Gegenden der Tundrajuraken ron einem Dialektgebiet ins andere kommen wollte. - Tंber seine Arbeit bei den Waldjuraken schreibt Lehtisalo: "Trotz einiger bedeutender, besonders lautlicher Unterschiedlichkeiten stellte ich fest, dass die waldjurakischen Dialekte sich sehr gleichen. Am interessantesten ist der östliche bzw. Pur-Dialekt, weil sich in ihm am meisten alte Züge bewahrt haben, die anderwärts geschwunden sind oder sich ausgeglichen haben. Leider hatte ich keine Gregenheit, ihn weiter zu erforschen; ich hätte eine Reise zu den östlichen Waldjuraken machen müssen, doch dazu reichte noine Stipendiumzeit nicht." Besonders bei den Waldjuraken musste Lehtisalo im Winter in den engen und unruhigen Behausungen der Samojeden arbeiten, wo er der Kälte wegen überhaupt nicht mit Tinte schreiben konnte. Da kein Tisch vorhanden war, musste er anstelle von losen Wortzetteln für die Aufzeichnungen Blöcke mit festen Deckeln verwenden, wobei es nicht selır leicht war, z.B. die Formen der rerschiedenen Dialekte miteinander zu vergleichen. Das Abschneiden derartiger Aufzeichnungen und das Kleben oder Kopieren auf Wortzettel musste dann nach der Reise in der Heimat geschehen. Das war eine schwierige Arbeit, die Jahre erforderte. Im Zusanmenhang damit zeigten sich auch einige Stellen, die zwecks İberprüfung und Kontrolle eine neue Sibirien-Reise erfordert hätten. In der Zeit nach der Oktoberrevolution in Russland bot sich jedoch finnischen Forschern bekannterweise keine Möglichkeit dazu. Eine bemerkenswerte Hilfe für Lehtisalo bedeutete jedoch der Tundrajurake Matvei Jaadnje, der im Sommer 1928 als Sprachmeister aus Leningrad zu ihm nach Finnland kam. Mit ihm arbeitete er konzentriert zweieinhalb Monate, wie aus jenem interessanten Bericht hervorgeht, der im JSFOu 44. veröffentlicht ist. Der 21jährige samojedische Studierende - ein früherer Rentierzüchter und Fischer - vermochte wichtige Angaben sogar über die Mythologie der Juraken zu machen. Ausserordentlich wichtig war, dass Lehtisalo gemeinsam mit einem beruflichen Phonetiker, Prof. Frans Äimä, die Artikulation seines Sprachmeisters im phonetischen Institut der Universität Helsinki gründlich erforschen konnte. Auf Grund seiner hierbei gemachten Beobachtungen hat er allem 
Anschein nach die Transkription seiner in Sibirien gemachten Aufzeichnungen in gewissem Masse ausgeglichen. Die Umschrift, die er u.a. in seiner jurakischen Volksdichtungssammlung (1947) und in seinem Wörterbuch (1956) verwendet, weicht nämlich in manchem von der i.J. 1927 in der Dissertation benutzten ab. Die spätere detaillierte Untersuchung der ausführlichen Feldaufzeichnungen verursachte sicher auch eine Reihe von Überprüfungen. Es erwies sich, dass auch einige andere Dinge der Vervollständigung bedurft hätten: "Die Kontrolle meiner Aufzeichnungen an Ort und Stelle wäre auch rom Standpunkt der Semasiologie nötig gewesen. Wenn ein Wort von irgendeinem sprachmeister eine andere Bedeutung erhalten hat, wäre es gut gewesen, auch anderswo zu fragen, ob diese abweichende Bedeutung bekannt ist oder ob sie sich vielleicht auf ein beschränktes Gebiet begrenzt. Ferner ist zu bemerken, dass das Fehlen eines Wortes im Wörterbuch für ein bestimuntes Gebiet oft nicht bedeutet, dass es dort unbekannt ist, sondern darauf beruht, dass ich nicht dazu kam, es zu erfragen».

Nach seiner Rückkehr von den Forschungsreisen hatte Lehtisalo leider nicht die wirtschaftlichen Möglichkeiten, sich mit voller Kraft dem Ordnen und Veröffentlichen seines umfangreichen wissenschaftlichen Materials zu widmen. Um sein Auskommen zu sichern, begann er zunächst an der Oberschule zu hospitieren, aber die Arbeit des Schullehrers lockte ihn jedoch nicht. Er zog bald aufs Land in die Gegend seiner Heimat. Seine Eltern und Geschwister waren in das Nachbarkirchspiel von Pöytyä gezogen, in das Dorf Kyrö der Gemeinde Karinainen, und diese kleine Ortschaft wurde für über zehn Jahre der ständige Wohnsitz des Samojedenforschers. Besonders in der Anfangszeit verliessen die Geldsorgen ihn nicht, weshalb Lehtisalo sogar ernsthaft überlegte, ob er nicht die kaufmännische Laufbahn einschlagen sollte. Die unsicheren Verhältnisse zur Zeit des Weltkrieges verhinderten jedoch - zum Glück -derartige Pläne, so dass er sich seinen eigenen Worten nach "dem Schicksal fügte und beschloss, die Arbeit auf seinem eigenen Gebiet fortzusetzen". Die anstrengenden Reisen hatten die Gesundheit des Forschers spürbar geschwächt, dem er dadurch abzuhelfen gedachte, dass er aktiv Sport trieb. In zwei 
drei Sommern tat sich Toivo Lehtisalo als $400 \mathrm{~m}$-Läufer in Sportwettkämpfen hervor. Es kam eine hübsche Sammlung von Preisen zusammen, doch die Hauptsache war, dass dank dem Sport die Gesundheit wiederhergestellt wurde. Die zweite Lieblingsbeschäftigung Lehtisalos war seit jenen Zeiten das Violinspiel, das ihn auch noch in seinem Alter erquickte. -- Die Hauptarbeit seiner Kyrö-Periode war ausser der erwähnten Wortschatzsammlung des Dialekts von Pöytyä die Vorbereitung seiner samojedischen Sammlungen zum Druck, da die Finnisch-ugrische Gesellschaft auf Initiative Kai Donners Mitte der 1920-er Jahre ein beständiges Arbeitsstipendium zu diesem Zweck bewilligt hatte. Zuerst bearbeitete Lehtisalo seine jurakischen Volksdichtungsproben. Die ursprünglichen Aufzeichnungen waren oft in grosser Eile gemacht, stellenweise sogar undeutlich, und der Forscher hatte bei weitem nicht alle Stellen an Ort und Stelle übersetzen können. Die Sprachproben umfassten an 730 Druckseiten, so dass bereits deren Vorbereitung zum Druck reichlich Zeit in Anspruch nahm, zumal da aus den Texten gleichzeitig weiteres Material für das Wörterbuch zusammenzustellen war. Auf den Reisen war das Hauptaugenmerk des Sammlers auf das lexikalische Material gerichtet gewesen, wobei er auch in phonetischer Hinsicht ausserordentlich genaue Arbeit leistete.

Vielfache Hindernisse verzögerten noch lange die endgültige Fertigstellung der Materialsammlungen von Lehtisalo, aber aus seiner Werkstatt - wozu ihm lange Zeit eine bescheidene Saunakammer diente - erschienen allmählich Untersuchungen und Werke, die sich in der Hauptsache auf sein eigenes samojedisches Material gründeten. Besonders die Fanggeräte und -methoden der Juraken schilderte er in seinem Artikel sZur jagd bei den juraksamojeden" (JSFOu 30:34), und in den Anfangsteilen von Kalevalaseuran vuosikirja (2, 3, 4, 7 und 9) erschienen interessante Schilderungen u.a. über die Totenverehrung der Juraken, über heilige Stätten und deren Geister sowie über Bogen, Pfeil und Zaubertrommel des juraksamojedischen Zauberers. Der als 53. Teil der MSFOu i.J. 1924 erschienene "Entwurf einer Mythologie der Jurak-Samojeden" ist eine auf eigenem Material des Verfassers wie in gewissem Masse auf schrift- 
lichen Quellen beruhende deskriptive Darstellung, wo die kosmogonischen Märchen der Juraken, die Geister des Himmels, der Erde und der Unterwelt, die heiligen Stätten und heiligen Tiere, die Zeltgeister, der Totenknlt und der Schamanismus behandelt werden. Für die Forscher der vergleichenden Religionswissenschaft hat sich dieses Buch als ausserordentlich wertrolle Quelle erwiesen. Die Vertreter der arktischen Sachvolkskunde wiederum haben dem 1932 in Oslo gedruckten Werk "Beiträge zur Kenntnis der Renntierzucht bei den Juraksamojeden" volle Anerkennung gezollt. Dessen 60 Seiten umfassender, nach Themenkreisen geordneter, terminologischer erster Teil enthält einen äusserst reichhaltigen Spezialwortschatz, der sich nicht bloss auf die Rentierzucht beschränkt, sondern z.B. auch die Ausdrücke für das Fahren mit dem Rentier, ja sogar auch den Wortschatz umfasst, der zu den transportierbaren Behausungen und deren Inventar gehört. Im übersichtlich gegliederten zweiten Teil des Werkes spricht Verf. u.a. über die Jagd des wilden Rens, über das Hüten der Rentiere, über die Wanderzüge der Nomaden und über die Produkte, die man vom Ren erhält. Ein eigenes Kapitel ist den urständigen Besitz- und Rechtsvorstellungen im Zusammenhang mit der Rentierwirtschaft gewidmet. Mit dem 23-seitigen Exkurs »Das Renntier in der Religion der Juraksamojeden" schliesst das Buch.

In einigen kürzeren Untersuchungen hat sich Lehtisalo mit weittragenden Fragen der geistigen Volkskunde und der Folkloristik beschäftigt. Auf dem Gebiet der vergleichenden Religionswissenschaft bewegt sich der im 14. Band (1934) des Kalevalaseuran ruosikirja gedruckte Aufsatz »Lepästä sielupuuna suomensukuisilla kansoilla" (¿̈ber die Erle als Seelenbaum bei den uralischen Völkern) und der recht umfangreiche Artikel "Der Tod und die Wiedergeburt des künftigen Schamanen" (JSFOu 48:3; 1937). Im letzterwähnten Aufsatz werden einige zur "Ausbildung" des Schamanenkandidaten gehörenden Auffassungen von den Samojeden, Jakuten, Burjaten, Tungusen, Giljaken, Eskimos, Indianern und Ureinwohnern Australiens geschildert. Mit fast ebenso ausgedehntem Vergleichsmaterial operiert Verf. in dem im Zusammenhang mit obigem veröffentlichten Aufsatz "Beobachtungen über die Jodlem" 
(JSFOu 48:2). Ausgehend von den lappischen, obugrischen und juraksamojedischen Jodlern werden darin u.a. die von den Schamanen verwendete heilige Geheimsprache, die "Sprache der Geister", und interessante Entstehungsfragen der Dichtung berührt. Lehtisalo besass umfangreiche Kenntnisse ïber die Folklore der verschiedensten Völker, und er beabsichtigte, seiner "Juraksamojedischen Volksdichtumg" ein vergleichendes universales Motivverzeichnis beizufügen. Zu diesem Zweck trieb er viel zeitraubende Detailstudien. Diese Vorarbeiten fanden sich in seinem umfassenden schriftlichen Nachlass, und deren Veröffentlichung wäre der vergleichenden Folkloristik sicher von Nutzen, wenn auch Lehtisalo selbst der Meinung war, die Zeit der vergleichenden Untersuchungen auf diesem Gebiet sei noch nicht angebrochen, da die Folklore der anderen Samojedenstämme und einiger Nachbarvölker vorläufig noch zu wenig oder überhaupt nicht bekannt ist (s. "Juraksam. Volksd.», Vorwort S. XI-XII).

Obwohl Lehtisalo viel Arbeit und Zeit auf die Erörterung ethnologischer Fragen verwandte, war die Sprachforschung doch sein eigentliches Gebiet. Das Thema für seine Dissertation wählte er aus der Vokalgeschichte des Samojedischen. Ende 1925 wurde in Kyrö das Manuskript zu diesem Werk fertiggestellt: "Über den vokalismus der ersten silbe im juraksamojedischen"; dessen Drucklegung in Deutschland beanspruchte jedoch wegen der fremden und komplizierten Transkription eine lange Zeit, so dass die akademische Disputation erst i.J. 1927 stattfand. Opponent war der zweite finnische Samojedenforscher Kai Donner, der anerkennende Worte über das Werk sprach, wenn er auch u.a. in einigen Vokalquantitätsfragen ganz anderer Meinung war als Verf. Das Hauptverdienst von Lehtisalos Dissertation war, dass sie viel neues, genau aufgezeichnetes Material aus dem Jurakischen enthielt. Verf. erstreckt seine Darstellung im Anhang seines Buches auf andere samojedische Sprachen und rekonstruiert den Vokalismus der ersten Silbe im Ursamojedischen. Dabei folgt er der von E. $\mathrm{N}$. Setälä dargestellten Ablauttheorie, die sich später als irrtümlich erwies. - Als erster Forscher wagte es Lehtisalo, die Geschichte des Vokalismus der gesamten uralischen Sprachfamilie 
in dem 50 Seiten umfassenden C̈berblick "Zur geschichte des vokalismus der ersten silbe im uralischen vom qualitativen standpunkt aus" (FUF 21; 1933) zu behandeln. In dieser Untersuchung wird zwar die gleiche, von Setälä angewiesene Erklärungsweise wie in der Dissertation angewandt, doch ist die Abhandlung dennoch in vieler Hinsicht weiterhin beachtenswert. - In einigen kleineren Artikeln hat Lehtisalo auch die Geschichte des Konsonantismus der uralischen Sprachen erläutert, u.a. brachte er eine eigene Theorie zur Entstehung des Stufenwechsels ror (Õpet. Eesti Seltsi Toim. 30; 1938).

Hinsichtlich der Quantität wie auch der Qualität ist Lehtisalos bedeutendstes sprachgeschichtliches Werk die 1936 erschienene 400 Seiten umfassende Untersuchung über die uralische Wortbildungslehre: "Über die primären ururalischen ableitungssuffixe" (MSFOu 72). Es handelt sich um das wichtigste Handbuch dieses Gebietes, nach dem sich die Forscher aller uralischen sprachen ständig orientieren. Das Werk ist das Resultat einer zehnjährigen Materialsammlung. Ausser der detailliert nach I,auten gruppierten systematischen Abteilung, wo für zahlreiche finnisch-ugrische Suffixe die Entsprechungen aus dem Samojedischen angeführt werden, hat das Buch eine interessante Einleitung. Darin wird u.a. die Entstehung der uralischen Flexionsendungen und suffixe aus den Pronomina erläutert, die sich hinsichtlich ihres Stammtypus eindeutig ron den Nomina und Verben unterscheiden. Bemerkenswert ist auch das Kapitel, wo der Vokalismus der zweiten Silbe der Wortstämme in der uralischen Ursprache erörtert wird. Neben der Lautgeschichte und der Wortbildungslehre beschäftigte sich Lehtisalo auch stark mit Fragen der Flexionslehre der uralischen Sprachen. In vielen Jahren behandelte er sie in seinen Vorlesungen an der Universität und sammelte auch von ausserhalb der uralischen Sprachen reichhaltiges Vergleichsmaterial.

Die Geschichte von reichlich hundert samojedischen Wörtern hat Lehtisalo im Laufe der Jahrzehnte in über zwanzig etymologischen Artikeln - in verschiedenen wissenschaftlichen Veröffentlichungen erschienen - erläutert; der letzte Aufsatz wurde in der Festschrift für Paavo Ravila in diesem .Jahr publiziert 
(MSFOu 125). Am umfangreichsten davon ist die Reihe "Uralische Etymologien" (MSFOu 58; 1928) mit 46 Wortvergleichen. Gewöhnlich geht er vom Juraksamojedischen aus, für dessen Wörter er besonders aus dem Obugrischen Entsprechungen anführt, oft auch aus dem Lappischen und mitunter sogar aus dem Ostseefinnischen. Bei seiner Erörterung über das Problem des Protolappischen stützte sich Y. H. Toivonen in ziemlichem Masse auf Lehtisalos Etymologien, und Björn Collinder hat sie recht zahlreich in den uralischen Abschnitt seines "Fenno-Ugric Vocabulary" aufgenommen. Es sei daran erinnert, dass gerade Lehtisalo als erster gezeigt hat, dass fi. neiti, pii und ylä bis ins ferne Ururalische zurückgehen und dass er auch an der Erklärung von sampo beteiligt war (Virittäjä 1929; vgl. weiter E. N. Setälä, Sammon arvoitus, S. 272). Seine im Druck erschienenen Wortvergleiche bilden jedoch nur eine bescheidene Auslese aus jener ausserordentlich reichhaltigen Sammlung etymologischer Aufzeichnungen, die hauptsächlich in den dreissiger und vierziger Jahren entstanden und nummehr archirmässig erfasst - an drei laufende Meter Wortzettel in Kleinformat darstellen. Er hatte sich in die vielfältigsten Sprachen und sprachfamilien vertieft und brachte in seinen Vorlesungen an der Universität in den 1940-er Jahren einige Ergebnisse seiner universalen Wortvergleiche zum Vortrag. Untersucht wurden damals auch sehr exotische Sprachen, bis zu den auf Feuerland, in Inner-Afrika und Polynesien gesprochenen Dialekten, obgleich die grossen Sprachgemeinschaften des Alten Kontinents und vor allem die nord- und mittelasiatischen Gruppen im Vordergrund standen, wenn von den uralischen Sprachen ausgehend - "Kulturwortausflüge" unternommen wurden. Er drängte jedoch nicht auf Veröffentlichung der Resultate seiner weitführenden Vergleiche, sondern war ihnen gegenüber später mitunter recht kritisch eingestellt.

Den unbestreitbaren Höhepunkt und die dauerhafte Basis von Lehtisalos schriftlichem Schaffen bilden seine stattlichen jurakischen Materialveröffentlichungen. Zu seinem 60. Geburtstag erhieit er die ersten Exemplare seines eben fertiggestellten Werkes "Juraksamojedische Volksdichtung" (MSFOu 90). 
Dessen über 600 Seiten enthalten u.a. jurakische Ursprungssagen, märchenartige Lieder und Erzählungen über die Entstehung der Geister, Sagen über Kämpfe zwischen Menschen, Riesen und bösen Geistern, Erwachsenen- und Kindermärchen, Berichte über die Wanderungen der Schamanen in jenseitigen Welten, Schamanenlieder, Zauberlieder und Opfergebete, all das mit genauen deutschen Übersetzungen. Die längste Sprachprobe in Versmass umfasst 1234 Strophen, und der umfangreichste Prosatext deckt 30 grosse Seiten. Das Werk stellt die überragendste samojedische Volksdichtungssammlung mit reichhaltigstem Inhalt dar. Es hat sich für die Samojedologen und die Uralisten als eine unersetzbare Schatzkammer erwiesen und kann auch den Folkloristen sowie vergleichenden Religionswissenschaftlern und Ethnologen sogar unerwartete neue Perspektiven eröffnen. Im Vorwort zu seinem Buch bringt der Verfasser interessante Bemerkungen über die Veränderlichkeit der schnellen Aussprache in den Jurakdialekten und deren Gründe. Über den dichterischen Wert der Folklore der Bewohner von Tundra und Taiga sagt er: "Ich kann nicht voraussehen, wie die besten juraksamojedischen Lieder einem solchen Leser vorkommen werden, der z.B. mit den formschönen und mit brennendem Gefühl verfassten indischen oder arabischen Märchen und Liedern vertraut ist. Demjenigen wenigstens, der mit den samojedischen Lebensverhältnissen vertraut geworden ist, bereiten sie einen ästhetischen Genuss, wenn er sie von einem Meister mit wunderbaren, fremdartigen Melodien gesungen oder mit tiefer Begeisterung rorgetragen hört.»

Als sein Hauptwerk hat Lehtisalo selbst sein "Juraksamojedisches Wörterbuch" bezeichnet, das erst 1956 erschien, obgleich das Manuskript - bis auf die Vorrede - zumindesten schon 1939 völlig druckfertig war. Die Kriegszeit, die anderen wissenschaftlichen Beschäftigungen des Verfassers sowie das mühsame Korrekturlesen verzögerten das Erscheinen dieses über 700 Seiten umfassenden grossen Werkes, das reichlich an 3500 Wortartikel enthält, viele von ihnen sehr umfangreich, durch charakteristische Beispielsätze veranschaulicht. Das ausführliche deutschsprachige Register erstreckt sich über 80 vierspaltige Seiten. Auch andere Forscher als die der samojedi- 
schen und uralischen Wortgeschichte finden in Lehtisalos Lexikon viel neues Material, aber bis jetzt haben z.B. nur ganz wenige Semantiker oder Ethnologen erkannt, ein wie wertvolles Hilfsmittel es bedeutet. Zwar mag die ausserordentlich genaue phonetische Transkription besonders beim Zitieren Schwierigkeiten bereiten. Das "Juraksamojedische Wörterbuch" ist insofern etymologisch, als darin angezeigt ist, welche Wörter von den nächsten Nachbarn, den Russen und Ostjaken entlehnt sind. Elemente russischen Ursprungs sind übrigens offensichtlich oft durch ostjakische Vermittlung in die sibirischen Dialekte des Jurakischen gedrungen. - Der eigentliche Wörterbuchteil des Werkes wurde von Prof. Gustav Schmidt ins Deutsche übertragen. Obgleich seine Übersetzung allgemein charakteristisch für ihn - gewandt und sorgfältig durchgeführt ist, lassen doch einige Einzelheiten darin zu wünschen übrig. Da die Ausdrücke des jurakischen Beispielmaterials aus der finnischen Übersetzung ins Deutsche übertragen wurden, wo das finnische Wort viele Bedeutungsnuancen haben kann, hat der Übersetzer eine solche Entsprechung wählen können, die die jurakische Wendung von der ursprünglichen wörtlichen Bedeutung recht weit entfernen mag. Die deutschen Übersetzungen der Ausdrücke und Wendungen sind also mitunter ziemlich frei - ohne jedoch jemals direkt fehlerhaft zu sein. Bei der Verwendung des Wörterbuches sollte man sich also über den wörtlichen Inhalt der Beispielsätze informieren, indem man die genaue Bedeutung eines jeden Wortes bei dem entsprechenden Stichwort sucht.

Noch i.J. 1960 erschien aus Lehtisalos fleissiger Feder die wichtige 463 Seiten umfassende Materialveröffentlichung "Samojedische Sprachmaterialien gesammelt von M. A. Castrén und T. Lehtisalo» (MSFOu 122). Hierin ist der letzte Teil seiner jurakischen Sprachproben enthalten. Darunter befinden sich Bruchstücke in Alltagssprache, die nicht zur Folklore gehören, Schilderungen vom Leben der Samojeden, z.B. über das Bauen eines Zeltes, über Nomadenwanderungen, über Jagd und Schleppnetzziehen sowie der Bericht über die Reise einer Samojedenfamilie nach Deutschland, wo die Tundrabewohner u.a. im Hamburger Zoologischen Garten als Wunder des Nordens 
gezeigt wurden. Das Buch enthält 26 Nummern eigentliche jurakische Folklore, insgesamt an 90 Seiten: zwei epische Lieder, zwei längere Sagen, 21 Schamanenlieder und ein Rauschlied. - Den Hauptteil des Werkes bilden Castréns selkupische und waldjurakische Aufzeichnumgen - Wörterverzeichnisse und Entwürfe einer Sprachlehre - , genau den ursprünglichen Manuskripten nach veröffentlicht. - Als Lehtisalo i.J. 1957 Leningrad besuchte, interviewte er ein selkupisches Mädchen, die dort studierte; die damals entstandenen Aufzeichnungen erschienen in diesem Buch gedruckt. - Lehtisalo hatte der uralischen Sprachwissenschaft und der Folkloristik ebenfalls einen wertvollen Dienst geleistet, als er i.J. 1940 das von Castrén aufgezeichnete jurakische und selkupische Volksdichtungsmaterial in der Gesamtheit (375 Seiten) als "Samojedische Volksdichtung" (MSFOu 83) herausgab.

Lehtisalo hat in seinen Materialveröffentlichungen genau das Bild bewahrt, das die urständige arktische Lebensform der Juraksamojeden noch vor einem halben Jahrhundert bot und das in einigen wesentlichen Zügen zum Lntergang verurteilt und stellenweise auch bereits schon geschwunden ist. So ist es offensichtlich, dass die Volksdichtung auf den Tundren und in der Taiga der Juraken nicht mehr in der Art und ebenso vielfältig blüht wie zu der Zeit, da der finnische Forscher dort wanderte. In den Rentierkolchosen und den neuen Kulturhäusern sowie in den Schulen und Klubs der Samojeden werden heute ein anderer Zeitvertreib und eine andere Dichtung gepflegt.

Toivo Lehtisalos schriftliches Schaffen ist Achtung gebietend umfangreich. Es umfasst weit über 3500 Druckseiten, davon über 2000 Seiten wichtige Materialsammlungen, von ihm selbst und von M. A. Castrén gesanmelt. Der Forscher hinterliess ausserdem zahlreiche Entwürfe und vergleichende Aufzeichnungen, die noch nicht zu Werken vervollkommnet waren, die aber wenigstens zum Teil binnen kurzem auf die eine oder andere Weise zum Nutzen der Uralistik, Folkloristik und Ethnographie verwertet werden dürften. Im Laufe der Jahre erfüllte Lehtisalo völlig jene Hoffnungen, die man in den ersten Jahrzehnten unseres Jahrhunderts auf ihn gesetzt hatte. Sein 
Aufstieg zum international anerkannten Wissenschaftler, zum führenden Samojedologen unserer Zeit, geschah ohne öffentliche Aufmerksamkeit zu erwecken, während er isoliert auf dem Lande lebte. Lehtisalos Wohnorte waren, nachdem er in seinen letzten Jahren in Kyrö eine Familie begründet hatte, Lavansaari, Säiniö und Selänpää sowie erst seit 1948 Helsinki. Von der Provinz aus versah er lange Zeit seine akademische Lehrtätigkeit: er las zwei Stunden in der Woche an der Universität Helsinki, wo er i.J. 1932 zum Dozenten für samojedische Sprach- und Volkskunde ernannt worden war.

Seine Universitätsvorlesungen bereitete Lehtisalo sorgfältig vor und er hatte seiner Zuhörerschar immer Neues zu bieten; sie war zwar zahlenmässig klein, aber um so dankbarer, sogar mitunter anspruchsvoll. So fragte der estnische Lektor Dr.Grünthal, Lehtisalos alter Freund und treuer Hörer, sehr oft während der Vorlesung nach dem Ursprung dieser oder jener samojedischen Wörter oder Formen. Die Vorlesung des Dozenten erinnerte manchmal an eine Seminarübung der Uralistik, wo diskutiert, ja sogar eifrig disputiert wurde. Viele ausländische Finnougristen nutzten während ihres Aufenthalts in Helsinki die seltene Gelegenheit und nahmen an den Vorlesungen des fimnischen Spezialisten für Samojedisch teil. In dieser gesamten Zeit ron einem Vierteljahrhundert (bis zum Jahre 1957), während der Lehtisalo Dozent war, sprach er in seinen Vorlesungen nur über Samojedologie; auch für die oben erwähnte, den ganzen Erdball umfassende Untersuchung ron Kulturwörtern bildete immer das Samojedische den Ausgangspunkt. Begründeterweise betonte er oft die Bedeutung der Kenntnis der entferntesten uralischen Sprachen für die Klärung der frühesten Phasen des Finnischen und bedauerte, dass dem Samojedischen innerhalb des Studiums der Finnougristik in Finnland kein bemerkenswerterer Platz eingeräumt war, ganz zu schweigen von den Prüfungshestimmungen für Finnisch und die ostseefinnischen Sprachen.

Als Toivo Lehtisalo 67 Jahre wurde, erhielt er den Titel und Rang eines Professors. Früher, kurz vor seinem 60. Geburtstag, hatte er diese Ehrenbezeigung abgelehnt, da noch Hoffnungen bestanden, dass er auf beständigere Weise dem als Dozent 
dem Lehrerkollegium der Universität Helsinki eingegliedert werde. Die Misserfolge innerhalb seiner akademischen Laufbahn erstickten zum Glück nie seinen Forschereifer, wenn sie ihn verständlicherweise auch verbitterten. Dieser ruhige und aufrichtige Gelehrte zeigte Aussenstehenden gegenüber eigentlich nicht seine Enttäuschungen, vielmehr war er in bekannter Gesellschaft ein fröhlicher Plauderer und Geschichtenerzähler. In den nachgelassenen Bruchstücken einer Autobiographie, die offensichtlich veröffentlicht werden sollten und um das Jahr 1960 entstanden sein mögen und die u.a. Kindheitserinnerungen sowie Reisebeobachtungen aus Ungarn (1931), Leningrad (1935) und London (1937) enthalten, kommt deutlich zum Ausdruck, wie tief die ihm widerfahrene wirkliche oder eingebildete Hintansetzung ihn berührt hat. In diesen stellenweise bekenntnisartigen und weltanschaulichen Anmerkungen trifft er zweifellos das Richtige, wenn er sich selbst z.B. auf folgende Art schildert: "Ich bin ein zu Einsamkeit und Isoliertheit neigender, recht passiver Charakter, der mehr daran interessiert ist, die Ereignisse philosophisch zu beobachten als aktiv mitzukämpfen. Doch habe ich einige Ereignisse nicht ohne eine Reaktion meinerseits vorübergehen lassen; auch ein Hund fährt einen an, wenn man ihn schlägt. Aber ich habe ausserdem den schlimmen Fehler, dass ich zur Mystik neige und fürchte, dass es unglücklich ausgeht, wenn man versucht, sich durch Falschheit Vorteile zu verschaffen. Und ich habe im Leben manches gesehen, was Vorsicht lehrt. - - Ein phlegmatischer Charakter, für den in $\mathbf{n}$ e r e Entwicklung und Weisheit das Wichtigste sind, wird hintangesetzt, ihm mag aber ein gutes Gewissen zum Lohn bleiben.»

Offensichtlich kurz bevor eine schwere Erkrankung im letzten Frühjahr ihn zwang, sich ins Krankenhaus zu begeben, schrieb Toivo Lehtisalo die folgenden Zeilen, die sich auf seinem Arbeitstisch fanden:

"Wenn der Mensch alt wird, zieht er sich allmählich praktisch und auch geistig aus dem Treiben der Welt zurück, vertieft sich nur in sich selbst und nähert sich einem vom Körper getrennten Leben. In dem Masse wie das erfolgt, fühlt er sich ruhig und glücklich. Die abstrakten Wissenschaften, wie die 
Mathematik, passen sich am besten in seine Gedankenwelt ein. Die Mathematik operiert mit Ziffern, Buchstaben, Formeln und Gleichungen. Aber auch Sprachwissenschaft lässt sich theoretisch betreiben, indem man mit den Lauten und deren phonetischen Zeichen operiert, mit aus ihnen gebildeten Silben, Wörtern und Sätzen. Es besteht nur der wichtige Unterschied, dass in der Sprachwissenschaft auch die Bedeutungen der Wörter unumgänglich sind. Die Sprachwissenschaft ist um so interessanter, je weiter sie abstrahiert werden kann. - - In der reinen Sprachwissenschaft sind die phonetischen Wahrnehmungen unbedingt notwendig. Ich habe von Sprachforschern gehört, dass die ganz genauen phonetischen Beobachtungen nicht nützlich sind, da dabei auch aufgezeichnet werden muss, was zur individuellen Aussprache des Sprachmeisters gehört und nicht typisch ist für alle, die den gleichen Dialekt sprechen. Bemerkenswert ist jedoch, dass man, wenn die Rede nicht genau aufgezeichnet wird, sondern Veränderungen vorgenommen werden, die der Aufzeichnende für nötig befindet, auf den Weg der Willkür und der Fälschung gerät, was bei der reinen Wissenschaft nicht angemessen ist. - - Ich kenne einen Fall, wo ein junger Ethnologe, als er einen heidnischen Friedhof fotografieren wollte, seine Gehilfen aufforderte, die oberirdischen Gräber in geraden Reihen anzuordnen, denn so würde das Foto seines Erachtens schöner. Die absichtliche Fälschung der Wahrnehmungen wie hier ist unbedingt zu verurteilen; das gleiche gilt für das Fälschen phonetischer Beobachtungen. Als ich als Student Beobachtungen über den Dialekt meiner Heimatgegend anstellte, hörte ich mir genau die Aussprache der verschiedenen Personen an. Dabei bemerkte ich, dass die Affekte, das Gefühl, das Zaudern, der Wunsch zu Übertreibungen u.dgl. in erstaunlichem Mass die Aussprache der gewöhnlichen Menschen beeinflussen. Wenn ein Sprecher z.B. etwas sagt, dessen Wahrheit er bezweifelt, kann er den kurzenVokal der ersten Silbe des Wortes dehnen, woraus der Hörer sofort seine Schlussfolgerungen zieht. Es heisst, in den westfinnischen Dialekten gebe es keine mouillierten Konsonanten. Ich habe Frauen getroffen, in deren Rede ganz deutlich ein mouilliertes I auftrat. z.B. ol rai hullu [schweige, Verrückte(r)!]. Dieser 
Laut kann nur festgestellt werden, wenn genau und vorurteilsfrei Wahrnehmungen gemacht werden. Wenn der Wissenschaftler ein solches Material in die Hände bekommt, erfüllt ihn ein Gefühl der Sicherheit, er fühlt, dass er ein Iraterial erhalten hat, das nicht gefälscht ist. Auch die Rede eines Mannes kann etwas Abweichendes in der Aussprache enthalten, das auf seinen individuellen Charakter zurückgeht und von den anderen nicht verwendet wird, ja ihnen sogar fremd erscheint, z.B. ein interdentales $t$, das mit etwas Spirantengeräusch verbunden ist. Die genaue Beobachtung all dessen eröffnet neıe Perspektiven für die Untersuchung der Beziehungen zwischen der Psychologie und der Sprache. Das Wechseln des musikalischen Akzents gehört noch mehr und näher zu Affekt, Gefühl und individueller Charakterbeschaffenheit als es bei den phonetischen Ausspracheschwankungen der Fall ist." - Bis zum Schluss beschäftigten sich die rege grbliebenen Gedanken des alten Forschers mit zentralen Problemen der Sprachwissenschaft.

Professor T. Lehtisalos langes, allein der Wissenschaft gewidmetes und an Resultaten reiches Leben erlosch in Helsinki am 8. August 1962. 